\title{
Hochverehrter Herr Präsident!
}

\section{Sehr geehrter Herr Klare!}

Mit dem AbschluB und dem Erscheinen dieses Teils hat das Kollektiv der Forschungsstelle für Akademiegeschichte an der Akademie der Wissenschaften der DDR unter meiner Leitung seinen ersten großen Arbeitsauftrag erfüllt. Dies ist Anlaß, Ihnen, hochverehrter Herr Präsident, für Ihre stete Anteilnahme und persönliche Unterstützung zu danken, die Sie der Erarbeitung der dreiteiligen Geschichte der Berliner Akademie der Wissenschaften in der Zeit des Imperialismus gewährt haben.

Sie haben in Ihrem langjährigen wissenschaftlichen Schaffen jene Erfahrung gewonnen, die Sie auch die wissenschafts- und akademiegeschichtliche Forschung fördern ließ.

Als junger Chemiker stellten Sie Ihre wissenschaftliche Arbeit mit der Hinwendung zur Polymeren- und Kunstfaserforschung in den Dienst der Praxis. Damals erlebten Sie, wie unhaltbar die ïberkommene These ist, die Wissenschaft sei von der Politik unabhängig. Nach der Befreiung von der faschistischen Herrschaft bestätigte sich für Sie in dreijähriger Tätigkeit in der Sowjetunion, daß Wissenschaft und Technik nur unter progressiven Gesellschaftsverhältnissen im Interesse des menschlichen Fortschritts entwickelt werden können. Wenige Tage nach der Gründung der DDR zurückgekehrt, stellten Sie Ihre Erfahrungen und Kenntnisse als Leiter des Faserstoffwerkes „Wilhelm Pieck" in Schwarza sowie später in verantwortlichen Funktionen des Instituts für Faserstofforschung der Akademie der Wissenschaften in TeltowSeehof für den wissenschaftlichen Aufbau in der DDR zur Verfügung. Sie hatten fortan persönlichen Anteil am Aufschwung der Wissenschaft, über den Sie rückschauend feststellten: „Mit der Gründung der Deutschen Demokratischen Republik begann ein neuer Abschnitt der Wissenschaftsentwicklung in der DDR. Die Regierung der DDR erwies von der ersten Stunde ihres Bestehens an der Wissenschaft umfassende Förderung. Besonderes Augenmerk galt dem Ausbau des Forschungspotentials... Partei und Regierung nahmen aktiven Einfluß auf die Zielstellungen der Forschungsarbeiten. .."

Als Leiter der Forschungsgemeinschaft der naturwissenschaftlichen, technischen und medizinischen Institute unserer Akademie übernahmen Sie Ig6I eine besonders hohe Verantwortung. Aus unserer damaligen engen Zusammenarbeit als Vizepräsidenten kenne ich Ihr Bemühen um die planmäßige Entwicklung des an der Akademie konzentrierten, zunehmend erweiterten sowie untereinander und mit der gesamten Forschung der DDR verflochtenen Potentials. 
Als Präsident setzen Sie sich seit Juli I 968 an führender Stelle für die Lösung der großen Aufgaben ein, die der Akademie und der gesamten Wissenschaft der Republik beim weiteren Aufbau der entwickelten sozialistischen Gesellschaft gestellt sind. „Wenn die Akademie die ihr von der gesellschaftlichen Entwicklung zugewiesene Stellung in unserem Staatswesen erfüllen will, dann muB es unser Anliegen sein, aus einer Gelehrtengesellschaft mit zugeordneten Forschungseinrichtungen eine moderne Forschungsakademie zu schaffen. . “ Diese Feststellung, die Sie auf dem Leibniztag I968 getroffen haben, hat richtungweisende Bedeutung für die Entwicklung der Akademie in der Gegenwart. Damit wurde es auch möglich, weiterführende konzeptionelle und methodologische Orientierungen für die Analyse der Vergangenheit, für die akademiehistorische Forschung zu gewinnen.

Das Postulat von Gottfried Wilhelm Leibniz als des Gründers und ersten Präsidenten der Akademie, die Wissenschaft mit der Praxis zu verbinden, erlangte angesichts der neuen Forderungen der sozialistischen Gesellschaft an die Wissenschaft in diesen Jahren Ihres Wirkens an der Spitze der Akademie qualitativ höhere Bedeutung. Damit waren auch für die Erarbeitung der Geschichte unserer Akademie neue Maßstäbe gesetzt.

Die Stellung der Wissenschaft in der modernen Welt, ihre Funktion in der Auseinandersetzung des Sozialismus mit dem Imperialismus und die Gefahr ihres destruktiven Mißbrauchs veranlassen Sie als Präsidenten immer wieder, gemeinsam mit den Führungsgremien der Akademie auf die gesellschaftliche Verantwortung des Wissenschaftlers hinzuweisen und dessen Verpflichtung zu unterstreichen, mit allen arbeitenden Menschen für die Nutzung der Wissenschaft im Dienste des Friedens, des Fortschritts und des Wohls der Völker zu kämpfen. Für die wissenschafts- und akademiegeschichtliche Forschung bestätigt sich in dieser Maxime das marxistisch-leninistische Prinzip, Gelehrte nach ihrer wissenschaftlichen Leistung einzuschätzen und dabei gleichzeitig ihr gesellschaftliches Denken und Handeln zu berücksichtigen.

Die Akademie kann den an sie gerichteten Anforderungen nur entsprechen, wenn sie ihre gesamte Arbeit auf den Marxismus-Leninismus gründet und insbesondere die disziplinäre und interdisziplinäre Kooperation ausbaut. Sie, Herr Präsident, orientieren stets nachdrücklich auf diese integrative Funktion, auf die Verantwortung der Akademie für das Zusammenwirken von Natur- und Gesellschaftswissenschaften, das erst mit dem umfassenden Aufbau der sozialistischen Gesellschaft ein reales Ziel darstellt. Hierbei geht es letzlich um jene Einheit der Wissenschaften, die, von vorausschauenden Gelehrten seit Jahrhunderten gefordert und angestrebt, in der Geschichte der Wissenschaft und unserer Akademie ihren Platz hat.

Die Aufgaben der Akademie vor allem in der Grundlagenforschung stellen an das Schöpfertum, die Einsatzbereitschaft und die Arbeitsweise ihrer Mitglieder und Mitarbeiter hohe Anforderungen. Die Rückbesinnung auf die Geschichte unserer Akademie, auf die Kreativität, das Wissenschaftsethos und den fruchtbaren Arbeitsstil ihrer führenden Mitglieder der Vergangenheit, ist auch in diesem Zusammenhang ein Kraftquell, auf den Sie immer wieder aufmerksam machen. Am Beispiel richtungweisender Chemiker wie Emil Fischer und Jacobus Henricus van't Hoff haben Sie dies u. a. in Ihrem wissenschaftshistorischen Vortrag vor dem Plenum der Akademie verdeutlicht.

Ihr Wirken als Wissenschaftler, hochverehrter Herr Präsident, fällt vor allem in jene historischen Jahre, in denen der Bruderbund zwischen den sozialistischen Staaten 
zu einer bewegenden und bestimmenden weltpolitischen Kraft wurde und in denen die Wissenschaftskooperation mit der UdSSR und den anderen Partnern der sozialistischen Gemeinschaft einen entscheidenden Aspekt der sozialistischen Wissenschaftspolitik von Partei und Regierung darstellt. Als Präsident haben Sie an der Entwicklung der wissenschaftlichen Zusammenarbeit mit der Sowjetunion und den anderen sozialistischen Ländern, an der Festigung der internationalen Stellung der Akademie hohen Anteil. Dies bestärkt uns Historiker in dem Bemühen, die wissenschaftsgeschichtlichen Traditionen, insbesondere die bewährte Zusammenarbeit zwischen deutschen, russischen und sowjetischen Gelehrten, zwischen der Wissenschaft in der DDR und in der UdSSR für jene Aufgaben fruchtbar zu machen, die der Akademie heute gestellt sind.

So empfängt auch die akademiegeschichtliche Forschung wertvolle Anregungen aus der Gestaltung unserer sozialistischen Gegenwart, der sie mit ihren Ergebnissen dienen möchte.

Die dreiteilige Darstellung der Geschichte der Berliner Akademie der Wissenschaften in der Zeit des Imperialismus wurde in den Jahren Ig68 bis I978 erarbeitet. Dem Präsidenten der Akademie, Herrn Professor Dr. Dr. h. c. Hermann Klare, darf sie zu seinem Ehrentage am I2. Mai I979, seinem 70. Geburtstag, in Dankbarkeit für stete verständnisvolle Unterstützung und vielfache Anregungen gewidmet werden.

Leo Stern 
